\title{
Dimensiones de las competencias científicas esbozadas en los programas de estudio de Biología, Física y Química de la Educación Diversificada y su relación con las necesidades de desarrollo científico-tecnológico de Costa Rica
}

\author{
Aspects of Scientific Competences Outlined in High School Biology, Physics and \\ Chemistry Syllabus and its Relation with the Scientific and Technological Development \\ Needs of Costa Rica
}

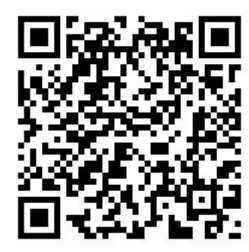

\author{
Cristina Padilla-Canales ${ }^{1}$ \\ Universidad Nacional \\ Heredia, Costa Rica \\ mcristina_padilla@hotmail.com \\ Paola Brooks-Calderón ${ }^{2}$ \\ Universidad Nacional \\ Heredia, Costa Rica \\ paubrooks@hotmail.com \\ Luis Diego Jiménez-Porras 3 \\ Universidad Nacional \\ Heredia, Costa Rica \\ mm3die@gmail.com \\ María Isabel Torres-Salas ${ }^{4}$ \\ Universidad Nacional, \\ Centro de Investigación y Docencia en Educación \\ Heredia, Costa Rica \\ isabeltorresr@yahoo.com
}

Recibido 23 de setiembre de 2014 • Corregido 31 de agosto de 2015 • Aceptado 17 de noviembre de 2015

\footnotetext{
${ }^{1}$ Bachiller en la Enseñanza de las Ciencias y Licenciada en Enseñanza de las Ciencias Universidad Nacional de Costa Rica.

2 Bachiller en la Enseñanza de las Ciencias y Licenciada en Enseñanza de las Ciencias Universidad Nacional de Costa Rica.

${ }^{3}$ Bachiller en la Enseñanza de las Ciencias y Licenciado en Enseñanza de las Ciencias Universidad Nacional de Costa Rica.

${ }^{4}$ Académica de la División de Educología, Universidad Nacional de Costa Rica Máster en Psicopedagogía, Bachiller en Ciencias de la Educación, Bachiller en la Enseñanza de la Química y Licenciada en Enseñanza de la Química.
} 
doi: http://dx.doi.org/10.15359/ree.20-1.2

URL: http://www.una.ac.cr/educare

CORREO: educare@una.cr

Resumen. El desarrollo científico y tecnológico de un país está directamente relacionado con el fortalecimiento de la educación en ese campo y con la capacidad de gestar el avance desde las bases mismas del sistema educativo. En este escenario, se consideró importante conocer si las políticas de desarrollo científico y tecnológico de Costa Rica guardan relación con los programas de estudio de la educación secundaria en la rama científica. Para ello, se realizó una investigación cualitativa utilizando el tipo de estudio hermenéutico. Los participantes fueron nueve docentes ( 3 de Biología, 3 de Física y 3 de Química) de colegios en instituciones de tres modalidades: académica, científica y técnica, así como personal catalogado como experto de las siguientes entidades: Ministerio de Ciencia y Tecnología, Consejo Nacional para Investigaciones Científicas y Tecnológicas, Estrategia para el siglo XXI, Academia de Ciencia, Centro de Comercio Exterior, INTEL y asesorías de Biología, Física y Química del Ministerio de Educación Pública. Las técnicas utilizadas para recolectar la información fueron la observación, el análisis de contenido, y la entrevista. Entre los principales resultados obtenidos se encontró que el programa de estudio de Biología es el que en mejor medida logra demostrar las dimensiones (conocimiento del saber y del saber hacer, capacidad, actitud y contexto) de las competencias evidenciadas a través de las capacidades esperadas a corto plazo, como son los objetivos, de los cuales se desprende la articulación de estas dimensiones en cada unidad temática del programa de estudios, aspecto determinante para que la educación media contribuya con el desarrollo científico y tecnológico del país. Además, en términos generales, se halló que dentro de las aulas existe una descontextualización entre lo que se imparte y el desarrollo que persiguen las políticas en este campo, porque hay una desvinculación entre el currículo pretendido en el programa de estudio y las políticas de desarrollo científico-tecnológico propuestas en el país.

Palabras claves. Desarrollo científico y tecnológico, políticas, competencias científicas, conocimiento, capacidad, actitud, contexto y currículo.

Abstract. The scientific and technological development of a country is directly linked to the strengthening of education in that field and the ability to brew advancement from the foundations of the education system. In this scenario, it was considered important to know whether the policies of scientific and technological development of Costa Rica relate to high school syllabus in the scientific area. Therefore, a qualitative research using a hermeneutic study was carried out. Nine participants (three biology teachers, three of physics, and three of chemistry) were selected from three kinds of educational institutions: academic, scientific and technical; as well as expert staff from the following entities: Ministry of Science and Technology, National Council of Scientific and Technological Research, Strategies for the 21st Century, Academy of Science, Center of Foreign Trade, INTEL and consultants of Biology, Physics and Chemistry from the Ministry of Public Education. The techniques used to collect information were: observation, content analysis and interviews. Among the main conclusions, it was found that biology syllabus is the one that best measures the dimensions (awareness of knowledge and knowledge to do, ability, attitude and context) from the evidenced competences through short-term expected capabilities, such as objectives, which suggests the articulation of these dimensions in each subject unit syllabus, a determining aspect to make high school education contribute to the scientific and technological development of the country. In addition, it was found, in general, that inside the classrooms there is a decontextualisation between what is taught and the development pursued by the policies in this field, because there is no connection between the educational plan intended in the syllabus and the scientific and technological development policies proposed in the country.

Keywords. Scientific and technological development, policies, scientific competences, knowledge, skill, attitude, context and educational plan. 
doi: http://dx.doi.org/10.15359/ree.20-1.2

Las exigencias de la sociedad en el mundo actual van direccionadas al incremento del capital económico con el fin de posicionarse en los mejores puestos a nivel mundial; para lograr este objetivo no solo se hace necesario que la ciudadanía maneje algunos elementos básicos relacionados con el lenguaje y las letras, sino que resulta trascendental el hecho de haya podido desarrollar conocimientos en el campo de la ciencia y la tecnología.

Estas disciplinas han tomado un importante auge en las últimas décadas en aquellas sociedades que han mostrado un desarrollo acelerado, en algunos casos ubicándose como naciones del primer mundo; en donde, el conocimiento científico ha trascendido prácticamente en muchos aspectos cotidianos y se ha vuelto indispensable no solo para la comprensión del medio en el que estamos inmersos, sino también para participar de manera fundamental en una sociedad democrática (González, M. T. Martínez, C. Martínez, Cuevas y Muñoz, 2009).

A través de la historia, el desarrollo científico-tecnológico de los diferentes países ha estado marcado por cambios importantes que se han gestado desde los currículos educativos. Tal y como lo menciona López (2006), el currículo es lo que configura la formación de los ciudadanos y ciudadanas de un país, sus competencias, su capacidad crítica para la percepción del mundo que les rodea, así como su capacidad para transformar la sociedad y adaptarse a los cambios, es decir, la educación siempre ha sido el principal eje movilizador de desarrollo científico-tecnológico de una nación. El Estado costarricense, representado principalmente por el Ministerio de Ciencia y Tecnología como ente rector en este campo, ha establecido políticas coherentes con las necesidades de desarrollo científico-tecnológicas del país acordes con las demandas nacionales e internacionales; sin embargo, no existe claridad en cuanto al nivel de relación de estas con los programas de estudio en esta área a nivel de secundaria.

En ese sentido se formuló la ley $N^{\circ} 7169$ que establece la promoción del desarrollo científico que sustenta la Fundación para el Desarrollo de la Ciencia y la Tecnología (CIENTEC) (Alfaro y Villegas, 2010); sin embargo, es un esfuerzo aislado que no ha sido suficiente, ya que no se reflejan cambios sustanciales en esta áreas De ahí la importancia de que los programas promovidos por el Ministerio de Educación Pública (MEP) gesten una base sólida en la educación científica que permita hacer frente a la globalización y a las tendencias de desarrollo científicotecnológico, guiadas en muchos casos por los mercados externos.

Por lo anterior, se consideró importante investigar acerca de las competencias científicas que se generan a largo plazo a raíz de la promoción de las dimensiones presentes en los objetivos de los programas propuestos para la educación diversificada en Biología, Física y Química; así como indagar, por medio de personas expertas en ciencia y tecnología, y en el ámbito educativo, sobre las políticas de desarrollo científico-tecnológico del país y su relación con los programas de estudio de la Educación Diversificada en este campo. 
doi: http://dx.doi.org/10.15359/ree.20-1.2

URL: http://www.una.ac.cr/educare

CORREO: educare@una.cr

\section{El estado de la cuestión}

Con respecto a los procesos educativos con énfasis en ciencia y tecnología Lemke (2006) investigó acerca del futuro de la educación científica y las nuevas formas de aprenderla, haciendo una reseña sobre el rediseño que debe sufrir la educación científica para encajar en el siglo XXI. Así mismo, Carrasco (2010) presenta una reseña sobre el libro Paradojas y dilemas de las universidades Iberoamericanas ante la sociedad del conocimiento de Adriana Gewerc Barujel, en el cual se analiza, de manera crítica y compleja, la formación del profesorado universitario con relación con las necesidades actuales de ciencia y tecnología.

En esta misma línea el Plan Nacional de Ciencia, Tecnología e Innovación 2011-2014, desarrollado por el Ministerio de Ciencia y Tecnología (MICIT) (2011), esboza una Costa Rica inmersa en los marcos de desarrollo tecnológico y describe la importancia de invertir en ciencia, tecnología e innovación para fortalecer el desarrollo del país, el cual ha venido acrecentándose en los últimos años. Así mismo menciona que el desarrollo científico-tecnológico se logra mediante el fomento de la educación científica que permita incentivar a las personas en la incursión de estudios universitarios que comprendan las ciencias y las tecnologías.

Referente al desarrollo de competencias científicas, Zúñiga, Leiton y Naranjo (2011), en Costa Rica y Argentina, llevaron a cabo la investigación Nivel de desarrollo de las competencias científicas en estudiantes de secundaria de (Mendoza) Argentina y (San José) Costa Rica, la cual tuvo como fin redefinir los objetivos y contenidos implementados en el currículo educativo, reorientándolos hacia un aprendizaje contextualizado del conocimiento científico que permita el desarrollo de competencias relevantes para todas las personas. Entre los principales resultados obtenidos se encontró que el estudiantado tiene gran capacidad para dar explicación a los fenómenos mediante la conceptualización científica; no obstante, la forma en que se imparte la educación tiene una gran tendencia a conseguir el aprendizaje de contenidos conceptuales, antes de los contenidos actitudinales o procedimentales.

En el 2009, se llevó a cabo en Bruselas el congreso internacional sobre las competencias del siglo XXI donde se dio lectura del informe Habilidades y competencias del siglo XXI para los aprendizajes del nuevo milenio en los países de la OCDE (Instituto de Tecnologías Educativas, 2010). Una de las principales conclusiones se refiere a la pretensión de reformar las escuelas y la educación para dar respuesta a las necesidades sociales-económicas de estudiantes y de la sociedad del siglo XXI. Es decir, la educación debe propiciar competencias acordes con las políticas de desarrollo de un país. 
doi: http://dx.doi.org/10.15359/ree.20-1.2

\section{Referentes conceptuales}

\section{Educación científica-tecnológica}

La educación científica, al ser uno de los ejes principales en el desarrollo socioeconómico de un país, requiere un replanteamiento integral para atender las demandas de la humanidad, a causa de la globalización que enmarca las nuevas necesidades y aspiraciones del ser humano en la búsqueda de un mejor desarrollo. No obstante, este desarrollo busca la integración de las competencias científicas y tecnológicas con la finalidad de subsanar las necesidades existentes en las áreas de la ciencia y la tecnología.

De acuerdo con Osborne y Dillon (2008); Rocard et al, 2007 (citados por García-Carmona y Criado, 2010), la educación científica debe contribuir a que el estudiantado adquiera una serie de competencias básicas, que le permitan desenvolverse con conocimiento, responsabilidad y espíritu crítico. Pero esas competencias que favorecen el desarrollo de las sociedades deben estar en concordancia con las principales tendencias de desarrollo de una nación, que según el informe realizado por el Ministerio de Educación de España (Ministerio de Educación, 2009). deben corresponder con las necesidades y requerimientos de la sociedad del siglo XXI; reflejar el estilo de pensamiento y acción de las ciencias de sujetos expertos; y adecuarse a las necesidades e intereses de los individuos beneficiarios.

Por ello, la educación científica y tecnológica cumple un rol fundamental en el desarrollo socioeconómico y cultural de un país, como lo cita Guadamuz (2005):

la Educación no puede estar ausente del proceso y del esfuerzo nacional [que se debe] realizar [para insertarse en las demandas y exigencias de un] mundo globalizado, [y así] producir la riqueza requerida para atender el desarrollo humano ..., para mantener los indicadores históricos, pero también, para mejorar significativamente los déficit y carencias aún existentes. (p. 2)

\section{Desarrollo científico-tecnológico de un país}

Para que haya desarrollo científico y tecnológico los países deben estar conscientes de que las reformas tienen que responder no solo a las necesidades actuales, sino también a las que continuarán apareciendo, ya que están inmersos dentro de un sistema de alta demanda y poca adaptación. Por ello, los países están obligados apostar a una reforma de la educación científica que no solo genere conocimiento, sino también que pueda satisfacer las necesidades de un modelo de producción.

Enfocándonos en Costa Rica, la inversión que se ha realizado en investigación, desarrollo, ciencia y tecnología se da mayoritariamente en el sector público, principalmente en las universidades (Ciarli y Giuliani, 2005). Sin embargo, se hace hincapié en que esta se encuentra muy por debajo de otros países de América Latina y más distante aún de los países desarrollados. 
doi: http://dx.doi.org/10.15359/ree.20-1.2

URL: http://www.una.ac.cr/educare

CORREO: educare@una.cr

Según Macaya (2006), "es estratégico conocer la situación del recurso humano existente en la región y sobre todo en la toma de decisiones sobre las orientaciones de la formación en recursos humanos... acorde con las necesidades y áreas potenciales para la mejora y competitividad de la región" (p. 12). Para ello, el país cuenta con el apoyo de universidades y en menor medida con el apoyo de empresas privadas que brindan algún tipo de formación. A nivel de recurso humano, en la formación de educación secundaria solamente se pueden mencionar los colegios técnicos profesionales y los científicos, en los cuales se fomenta, de forma clara, la inclinación del estudiantado hacia carreras relacionadas con ciencia y tecnología. Sin embargo, no hay claridad en cuanto al nivel de coordinación entre los diferentes entes del Estado, MICIT, MEP, CONICIT, universidades, entre otros, para que las políticas de desarrollo tengan concordancia con los programas de estudio del área científica y tecnológica en secundaria.

\section{Relación entre educación, ciencia y tecnología, y el desarrollo de un país}

La aplicabilidad de las ciencias y tecnologías forman parte de una amplia gama de actividades humanas que conllevan al desarrollo de los países $y$, por tanto, es fundamental comprender la importancia que arraiga una educación científica que permita alfabetizar a la sociedad mediante conocimientos científicos y tecnológicos, para atender el desarrollo humano, pero también mejorar significativamente los déficit y carencias aún existentes (Guadamuz, 2005).

Vivimos en un mundoen el queel conocimiento científico-tecnológicocondiciona su constante y rápida evolución, por lo tanto, resulta evidente que la educación científica cumple un papel muy importante en la sociedad, porque se necesitan una ciudadanía con más formación científica, capaz de tomar decisiones basadas en el conocimiento, así como personal científico en diferentes campos para responder a patrones complejos de articulación de acciones en los que diversos actores y visiones de mundo se entremezclan y conforman el desarrollo económico, científico, y tecnológico de un país (Alfaro y Villegas, 2010). Sin embargo, para que esto se lleve a cabo es necesaria una acción de cambio decisiva en el campo de la educación en ciencia y tecnología.

\section{Competencias científica-tecnológicas en la enseñanza de las ciencias}

La competencias científicas son definidas como aquellas capacidades que tienen los individuos para emplear el conocimiento científico, identificar preguntas y obtener conclusiones basadas en pruebas con el fin de comprender y ayudar a tomar decisiones sobre el mundo natural y los cambios que la actividad humana produce (Gobierno Vasco, Universidades e Investigación, 2011).

El desarrollo de las competencias depende de las características y finalidad del currículo, ya que existe diferencia entre las competencias que son dirigidas a la formación de individuos científicos y aquellas que buscan formar ciudadanos y ciudadanas con conocimiento en ciencia y tecnología_(Sanmartí, 2008).

6 
doi: http://dx.doi.org/10.15359/ree.20-1.2

Se puede entender por competencias científicas aquellas aptitudes que son enseñadas durante la formación escolar que le permiten eventualmente a la ciudadanía de una nación aplicar de forma razonable y crítica los conocimientos que fueron aprendidos para dar explicaciones acordes con los sucesos naturales, científicos y tecnológicos que ocurren en el medio socio ambiental en que se desarrolla. Además, es importante destacar que el conocimiento científico y tecnológico es un factor esencial en el desarrollo de las sociedades modernas.

\section{Diseño y metodología}

Esta investigación tuvo como objetivo estudiar si existe relación entre las políticas de desarrollo científico-tecnológico del país y las dimensiones (contexto, conocimiento del saber y del saber hacer, procedimientos y actitud) de las competencias científicas evidenciadas a través de las capacidades esperadas a corto plazo, como son los objetivos, de los cuales se desprende la articulación de estas dimensiones en cada unidad temática del programa de estudios de Biología, Física y Química de la Educación Diversificada del Ministerio de Educación Pública de Costa Rica.

Para identificar cuáles de las dimensiones de las competencias científicas mencionadas están presentes en los programas de Biología, Química y Física se tomaron los objetivos como ejes centrales de análisis, utilizando la técnica análisis de contenido, con el fin de conocer qué tanto se potencia el desarrollo de competencias científicas en el estudiantado del ciclo diversificado desde el área científica. Es importante aclarar que estos programas de estudio del Ministerio de Educación Pública no están planteados por competencias, porque para que un programa se fundamente en un enfoque de esta naturaleza, su base epistemológica y la forma en que se estructura el currículo debe reflejar la intencionalidad de formar por competencias. Sin embargo, en los objetivos de un programa de estudio, aún bajo otro enfoque se muestran dimensiones que potencian capacidades que contribuyen a la formación de competencias, de tal forma que se transforman en elementos de conocimiento a los cuales el individuo pueda recurrir para dar solución a problemas de la cotidianidad con conocimientos científicos.

En el análisis se manejó el criterio propuesto por la OCDE (2006), en el programa PISA donde hace mención a cuatro dimensiones que se detallan a continuación:

- Contexto: dentro de este se abarcan todas aquellas situaciones de la vida cotidiana donde existe un componente científico y tecnológico.

- Conocimiento del saber: abarca todos aquellos hechos donde se pueda comprender el mundo natural por medio del conocimiento científico en el que incluye el conocimiento del mundo natural como el de la propia ciencia.

- Capacidades: son acreditadas por las habilidades que se tienen frente a una ciencia, tales como identificar, explicar y extraer conclusiones científicas.

- Actitudes: son reconocidas por el interés, motivación y respaldo hacia la ciencia. 
doi: http://dx.doi.org/10.15359/ree.20-1.2

URL: http://www.una.ac.cr/educare

CORREO: educare@una.cr

En la tabla 1 se ejemplifica una de las unidades analizadas del Programa de Estudio de Química Educación Diversificada del MEP (2005, s. f.).

Tabla 1

Dimensiones de las competencias científicas la Unidad: La materia base del Universo

\begin{tabular}{|c|c|c|c|c|c|c|c|}
\hline \multicolumn{8}{|c|}{ Unidad: La materia base del Universo } \\
\hline \multicolumn{8}{|c|}{ Dimensiones de las competencias científicas } \\
\hline \multicolumn{2}{|c|}{ Contexto } & \multicolumn{2}{|c|}{ Conocimiento } & \multicolumn{2}{|c|}{ Capacidad } & \multicolumn{2}{|c|}{ Actitud } \\
\hline \multicolumn{8}{|c|}{$\begin{array}{l}\text { Objetivo 1. Analizar la importancia de la Química respecto al desarrollo de procesos industriales, el avance en } \\
\text { la tecnología, los procesos biológicos y su impacto en el ambiente, la alimentación, la salud y el desarrollo } \\
\text { sostenible en general. }\end{array}$} \\
\hline Sí & No & Sí & No & Sí & No & Sí & No \\
\hline \multicolumn{8}{|c|}{$\begin{array}{l}\text { Objetivo 2. Describir las propiedades de las sustancias puras y mezclas, así como su importancia en el mejora- } \\
\text { miento de la calidad de vida. }\end{array}$} \\
\hline Sí & No & Sí & No & Sí & No & Sí & No \\
\hline \multicolumn{8}{|c|}{$\begin{array}{l}\text { Objetivo 3. Analizar las características de los elementos químicos y su incidencia en los diferentes procesos } \\
\text { biológicos, geológicos y químicos que ocurren en la naturaleza, en la industria y en la vida cotidiana. }\end{array}$} \\
\hline Sí & No & Sí & No & Sí & No & Sí & No \\
\hline \multicolumn{8}{|c|}{$\begin{array}{l}\text { Objetivo 4. Describir las principales partículas que constituyen el átomo y su relación con el número atómico, } \\
\text { número másico, isótopos y masa atómica promedio. }\end{array}$} \\
\hline Sí & No & Sí & No & Sí & No & Sí & No \\
\hline
\end{tabular}

Nota: Elaboración propia a partir del análisis del Programa de Estudio de Química Educación Diversificada (MEP, s. f.).

Además, para conocer qué ocurre en relación con las dimensiones: contexto, conocimiento, capacidad y actitud al poner en práctica los programas del área científica, se realizaron una serie de observaciones a 3 profesores de Biología, 3 de Física y 3 de Química en colegios de distintas modalidades: colegio académico, científico y técnico, esta muestra fue seleccionada de manera aleatoria, lo que si fue intencionado fue la modalidad de la institución. Para recolectar la información de esta población se utilizaron escalas de apreciación gráfica que agruparon una serie de características por dimensión, indicando si los docentes las promovían, para ello se utilizó la categoría siempre, a veces o nunca y, por último, se trabajó con la técnica de entrevista semi-estructurada, dirigida tanto a personas expertas en ciencia y tecnología, como al ámbito educativo, para conocer sus opiniones acerca de las políticas de desarrollo científico- 
doi: http://dx.doi.org/10.15359/ree.20-1.2

tecnológico del país y su relación con los programas de estudio de la Educación Diversificada en el Área Científica. Es importante mencionar que todos los instrumentos utilizados para recabar la información fueron validados por el criterio de personas expertas con conocimiento en competencias científicas.

Los resultados se presentan en torno a tres categorías de análisis:

- Presencia de las dimensiones de las competencias científicas en los programas de estudio de Biología, Física y Química

- Dimensiones de las competencias científicas promovidas por el personal docente al impartir las disciplinas de Biología, Física y Química en colegios de tres modalidades distintas.

- Opinión de personas expertas sobre las políticas de desarrollo científico-tecnológico del país y la relación con los programas de estudio de la Educación Diversificada en el área científica.

\section{Resultados y discusión}

\section{Presencia de las dimensiones de las competencias científicas en los Programas de Estudio de Biología, Física y Química}

Los programas de estudio utilizados para la investigación son el de Biología que contiene 19 objetivos, el de Física con 21 y el de Química que contiene 30 objetivos. En el figura 1 se muestran los resultados que identifican cuáles dimensiones de las competencias científicas están presentes en los objetivos de los diferentes programas mencionados; se hace una comparación por dimensión.

Con respecto a la dimensión contexto (a), se puede afirmar que Biología es la de mayor presencia en comparación con Física y Química que solo alcanzan un 15\% y un $10 \%$ respectivamente. La contextualización es una herramienta importante que permite tanto a docentes como a estudiantes visualizar el entorno en que se desarrollan, como lo dice Ramsden (1988) citado por Salas (2008), el aprendizaje se desarrolla en diversos contextos educacionales, ya que dentro de estos se visualiza las vivencias del alumnado, lo que permite adaptarse a las exigencias y oportunidades presentadas por el contenido que tiene que ser aprendido en relación con su entorno institucional.

Por otro lado, cuando se analiza la dimensión de conocimiento (b), se encuentra que la mayor presencia de esta capacidad corresponde a Física con un 75\%, seguido de Biología con un $55 \%$ y Química con un 45\%, aspecto que es importante de tomar en cuenta, ya que 
doi: http://dx.doi.org/10.15359/ree.20-1.2

URL: http://www.una.ac.cr/educare

CORREO: educare@una.cr

$$
\text { B = Biología } \mathrm{F}=\text { Física } \mathrm{Q}=\text { Química }
$$
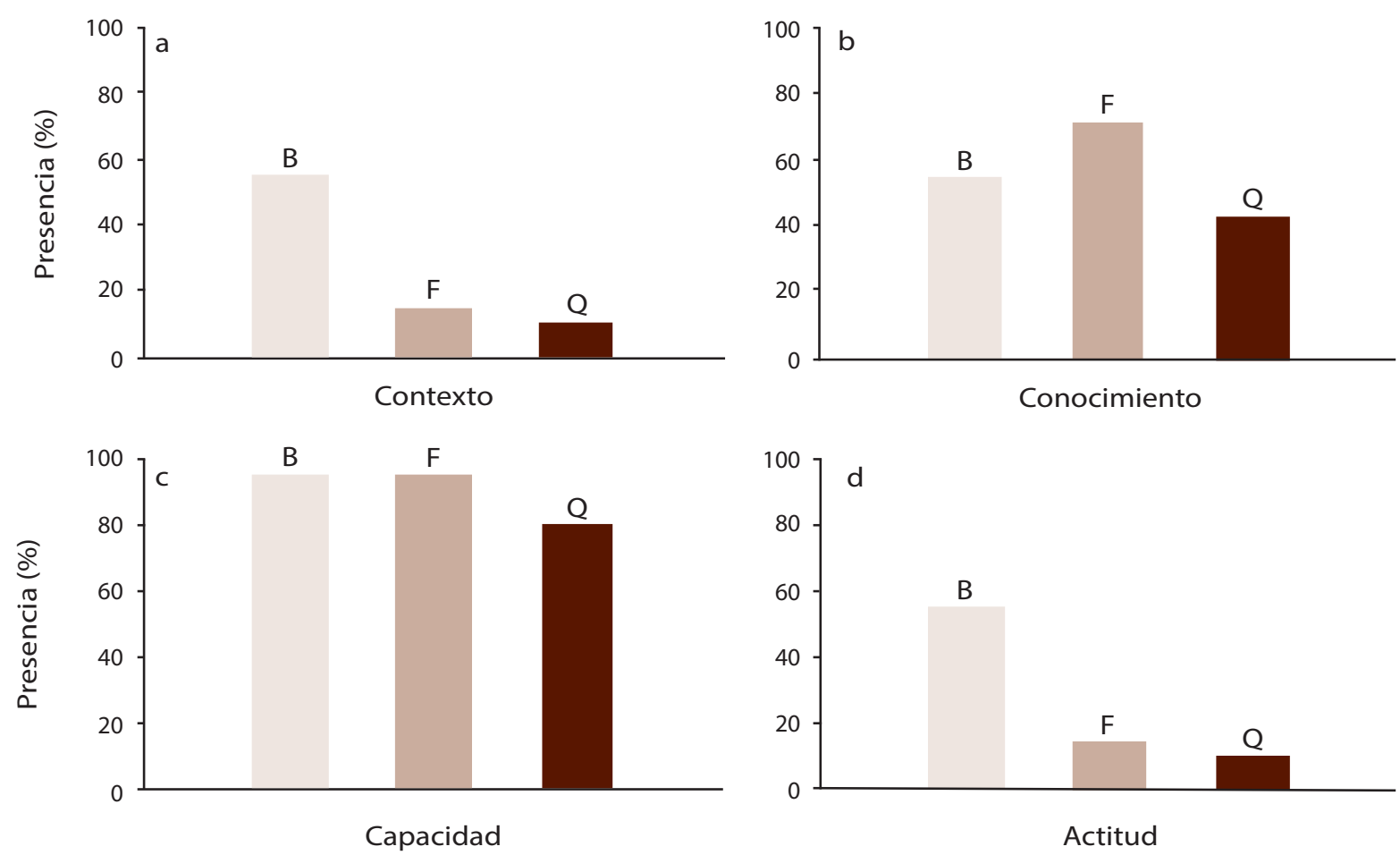

Figura 1. Dimensiones de las competencias científicas. Elaboración propia a partir de las que están presentes en los objetivos de los Programas de Estudio de Biología de Educación Diversificada (MEP, 2005), Programa de Estudio de Física, Educación Diversificada (MEP, 2003) y Programa de Estudio de Química, Educación Diversificada (MEP, s. f.).

sería de esperar que dicha dimensión estuviera explicita en un 100\% en cada uno de los objetivos, porque constituye el qué enseñar; sin embargo, al no estar planteados con un enfoque por competencias se comprende que las dimensiones no estén presentes en forma optima. Según el Ministerio de Educación (MEDUCA, 2012), cada asignatura, de acuerdo con su enfoque, presenta sus propios cánones de saberes conceptuales, permitiendo así saber distinguir y priorizar aquellos que son más útiles para el desarrollo de la competencia conforme el propio nivel educativo, los contextos y los tiempos, por lo que no debe estar ausente en un objetivo.

Referente a la dimensión de capacidad (c) presenta porcentajes muy altos y similares entre los tres programas, lo que resulta esperable si se busca acreditar la presencia de una serie de capacidades, como identificar cuestiones científicas, explicar fenómenos 
doi: http://dx.doi.org/10.15359/ree.20-1.2

científicamente y extraer conclusiones basadas en pruebas, evidenciando entonces que los programas de estudio propuestos por el MEP buscan más un aprendizaje basado en un conocimiento procedimental. Aspecto importante porque, de acuerdo con Jabif (2007), los métodos o las formas en que se realizan los procedimientos permiten la puesta en práctica del conocimiento.

Por último, la dimensión actitud (d), está presente en porcentajes muy bajos, como se puede ver en Química solo un 10\% y en Física un 15\%. Situación que llama a reflexionar porque el valor de la dimensión actitud es relevante, ya que, según Perrenoud (2004, citado por Cano, 2008), esta permite enfrentar eficazmente situaciones análogas, movilizando rápido, pertinente múltiples recursos cognitivos: saberes, capacidades, micro-competencias, informaciones, valores, actitudes, esquemas de percepción, de evaluación y de razonamiento.

En términos generales, de los programas analizados el de Química es el que presenta en menor medida las dimensiones de las competencias científicas, exceptuando la de capacidad y el de Biología es el que presenta las cuatro dimensiones en mayor medida y de manera más equitativa, presentando resultados por encima del $50 \%$ en todas, lo que evidencia que existen diferencias significativas en el planteamiento de los programas de estudio entre las distintas áreas.

Deacuerdocon lo encontrado podría pensarse que, a pesar de que estos programas no están planteados con un enfoque por competencias como ya se ha mencionado, resulta interesante saber que, al menos, se presentan las cuatro dimensiones, aunque no de forma equitativa. Con ello se espera, entonces, que los graduados y graduadas desarrollen algunas destrezas y habilidades que les permitan identificar, adquirir y explicar los sistemas y fenómenos naturales más relevantes, además comprender la forma en que el entorno condiciona las actividades humanas, las consecuencias de esas actividades en el medio ambiente, las aplicaciones y desarrollos tecnológicos de la ciencia, actuar consciente y eficazmente en el cuidado de la salud personal y extraer conclusiones basadas en pruebas sobre temas relacionados con las ciencias y su aplicación práctica en la vida cotidiana en la toma de decisiones (Gobierno Vasco, Universidades e Investigación, 2009).

Es importante aclarar que la presencia de las dimensiones en los objetivos son de gran trascendencia, porque estos aseguran el qué y cómo se deben enseñar los contenidos que involucran cada una de las unidades de los programas mediante acciones que faciliten el entendimiento y la comprensión de los fenómenos que incurren en el diario vivir; además de contextualizar los saberes permitiendo, entonces, la cercanía del estudiantado con la realidad científica-tecnológica del país. 
doi: http://dx.doi.org/10.15359/ree.20-1.2

URL: http://www.una.ac.cr/educare

CORREO: educare@una.cr

\section{Dimensiones de las competencias científicas promovidas por docentes al impartir las disciplinas de Biología, Física y Química en colegios de tres modalidades distintas}

Contexto: En la disciplina de Biología existe una escasa promoción de la dimensión contexto, siendo el educador del colegio académico el que menos fomenta la contextualización y el del colegio científico el que más lo hace. Esta dimensión tiene un papel muy importante en el aprendizaje, ya que entre otros aspectos, el no promoverla conlleva a que el estudiantado no encuentre relación entre lo que aprende y su entorno, además Caamaño (2005, citado por Pedrinaci, 2011) dice que, al contextualizar la ciencia, hay un momento del desarrollo del aprendizaje donde es relacionado con la vida cotidiana, lo que trae como consecuencia que se incremente el interés por la ciencia.

En esta misma línea, el personal docente del área de Física tiene una tendencia similar al de Biología, ya que la implementación de esta dimensión es escasa en los tres colegios. En cuanto al profesorado del área de Química fue variado, el educador del colegio técnico no implementa la contextualización dentro del desarrollo de las lecciones observadas, el docente del colegio académico lo hace poco y el profesor del colegio científico siempre fomentó esta dimensión en sus lecciones. En síntesis esta es una dimensión que el personal docente de las diferentes modalidades casi no promueve, además, desde los programas también se potencia poco.

Para implementar la contextualización de lo enseñado en el aula; de acuerdo con De Freitas Dias de Souza y Alves (2010), "la importancia de la contextualización de lo que se enseña (o aprende) [es] ... innegable. Es a través de este proceso como se puede transformar al conocimiento en algo placentero o al menos útil" (p. 278). A su vez, Hernández y Zacconi (2010) mencionan que el papel de quien educa debe encontrarse en primera instancia en desmitificar la ciencia y contextualizar las materias científicas a la vida diaria y su entorno, y además debe hacer de sus prácticas una tarea interesante y amena para atraer a los alumnos y alumnas a su aprendizaje.

Conocimiento: El personal docente de Biología de los tres colegios promueve la dimensión de conocimiento, de igual forma lo hacen los profesores de Física y los docentes de Química, esta dimensión es entonces la más promovida por los docentes observados. Se debe destacar que aunque no estaba presente en muchos objetivos, sí alcanza porcentajes altos en el desarrollo de las clases, situación que refleja que independientemente de la modalidad educativa el modelo de enseñanza que prevalece es el de la escuela tradicionalista, donde lo central es la trasmisión de conocimientos, modelo en el cual, según Ceballos (2004), el método de enseñanza es el mismo para todos los niños y niñas, y en todas las ocasiones. El aprendizaje es entendido como la repetición de lo que el profesorado dice.

Es importante aclarar que aunque los tres docentes están promoviendo esta dimensión de conocimiento, no implica que todo el estudiantado esté formando las competencias científicas para dar solución a problemas de la cotidianidad con conocimientos científicos. Porque es con 
doi: http://dx.doi.org/10.15359/ree.20-1.2

la incorporación de todas las dimensiones que se logra el desarrollo de una competencia, es decir, formar seres humanos "con ciencia, pero también con conciencia", afirmación de Morín (2007, citado por Torres, 2010). Esto, según Torres, constituye la principal tarea de la enseñanza de las ciencias contemporáneas.

Capacidad: En relación con la dimensión de capacidad, en el área de Biología, tanto el docente del colegio científico como el del técnico fomentan esta dimensión; caso contrario sucede con el del colegio académico que no la promueve. Asimismo, en el área de Física se pudo observar que los docentes del colegio académico y técnico no trabajan esta dimensión, lo que permite evidenciar que promueven poco el aprendizaje con base en los procedimientos. Por otra parte, el docente del colegio científico promueve en mayor medida esta dimensión con respecto a los profesores de los otros colegios.

En el área de Química, el docente del colegio científico promueve en gran manera una enseñanza procedimental, caso contrario ocurre con los docentes del colegio académico y técnico.

Para lograr la comprensión de lo que se pretende enseñar, indistintamente de la disciplina ejercida, los cuerpos docentes deben promover el conocimiento a base de procedimientos, esto porque, de acuerdo con Cordón (2008), además de facilitar que el estudiantado aprenda los contenidos conceptuales básicos que caracterizan a cada disciplina, la educación científica debe propiciar que adquiera habilidades y destrezas relacionadas con la actividad científica, desarrollando así valores y actitudes vinculados a la aplicación de la ciencia para la comprensión y resolución de diversas situaciones y problemas de la vida cotidiana, como los relacionados con la tecnología, la salud, el medio ambiente o el consumo. Aunque en los programas de estudio de las diferentes áreas esta es la dimensión con mayor presencia, en la práctica es la que menos se promueve.

Actitud: En cuanto a la competencia de actitud, en el área de Biología los tres docentes promueven esta dimensión, así mismo los del área de Física; sin embargo, el profesor del colegio académico no siempre lo hace. Los profesores del área de Química promueven esta dimensión de manera distinta, ya que docentes de los colegios científico y técnico sí la desarrollan, pero el docente del colegio académico casi no la promueve.

En términos generales, se puede afirmar que el personal docente consultado de Biología, Física y Química, de las modalidades académica, científica y técnica -a excepción del profesor de Química del colegio académico-, promueven el desarrollo de la dimensión de actitud. Aspecto que se considera positivo, ya que desarrollar este tipo de competencia es importante porque, según Arnau y Montané (2010, citando a Pérez, 2008), esta adquiere "cada vez más importancia para planificar la formación, evaluación, acreditación y ejecución de las profesiones, especialmente dentro de una sociedad basada en la información y en la cual existe la necesidad de orientaciones... holísticas que favorezcan el desarrollo del aprendizaje" (p. 1285). 
doi: http://dx.doi.org/10.15359/ree.20-1.2

URL: http://www.una.ac.cr/educare

CORREO: educare@una.cr

Curiosamente, la dimensión actitud es la que menos está presente en los objetivos de los programas de estudio, por lo que se podría esperar que no fuera promovida en el aula; no obstante, en la práctica se encontró lo contrario, incongruencias esperadas, ya que en la intencionalidad de los programas no está contemplado el trabajar las dimensiones, por lo que las prácticas docentes no responden a una concepción epistemológica que les oriente, sino más bien queda a criterio de cada quien promover, en su labor de aula, una u otra dimensión casi de una forma aleatoria.

\section{Opinión de personas expertas sobre las políticas de desarrollo científico-tecnológico del país y la relación con los programas de estudio de la educación diversificada en el área científica}

Sobre cómo califican el nivel de desarrollo científico - tecnológico del país, el personal entrevistado de COMEX, CONOCIT, fundación CIENTEC e INTEL concuerda que al realizar esta evaluación se deben tomar en cuenta dos criterios: El primero de ellos a nivel mundial, donde consideran que el desarrollo de Costa Rica se puede calificar como término medio, ya que faltan centros de investigación, y los que existen no abarcan todas las áreas de estudio. El segundo a nivel latinoamericano, donde ubican al país mejor posicionado, ya que se han tenido grandes avances en desarrollo científico y tecnológico con respecto a otros países de la misma región; sin embargo, agregan que aunque esto es alentador, aún no se cuenta con suficiente recurso humano para generar investigación en ciencia a nivel de empresas e instituciones públicas, ya que las personas que tienen doctorados en esta área se encuentran dentro de las universidades y los programas que se implementan no tienen suficiente proyección e impacto para el desarrollo del país, y esto se debe a que existe una desvinculación entre el sector productivo y la academia.

Cabe agregar que dos de los expertos mencionan que es difícil realizar esta evaluación, debido a que existen algunos aspectos en los que el país se encuentra muy bien, sin embargo, en otros se debe trabajar aún más. Esto se debe, en parte, a que la integración de la ciencia y tecnología en la sociedad no se desarrolla con la rapidez que un mundo globalizado exige. Además, para fomentar un desarrollo más integral en el área científica y tecnológica, se debe fortalecer el área de investigación en los aspectos referentes a inversión en recurso humano y en equipo, tanto en el campo de la ciencia como en la tecnología. Además, según el MICIT (2012): "la ciencia, la tecnología y la innovación son herramientas trascendentales ya que tienen una incidencia directa en el desarrollo económico y social de las naciones" (p. 17).

Acerca de las necesidades de desarrollo del país en estas áreas, la mayoría de las personas entrevistadas piensan que se deben incrementar las inversiones en infraestructura, lo cual es coherente con lo mencionado por el Instituto Tecnológico y de Estudios Superiores de Monterrey (2009), al decir que la inversión proporciona una base esencial para el desarrollo de las actividades económicas y, además, es una característica de las naciones o regiones subdesarrolladas la inexistencia de infraestructura en cantidad o formas necesarias, lo que constituye un obstáculo para su desarrollo. 
doi: http://dx.doi.org/10.15359/ree.20-1.2

Por otro lado señalan la falta de vocaciones científicas como un factor que incide directamente sobre el desarrollo de un país cuando de ciencia y tecnología se trata, mencionan que deben existir mecanismos para incrementar el recurso humano en estas áreas. La Organización de Estados Iberoamericanos para la Educación (OEl, 2012), dice que este problema se agudiza en las áreas de las ciencias exactas, naturales y en algunas ramas de las ingenierías fuertemente asociadas con las demandas actuales de la industria. Lo anterior se refleja directamente en la declive en la matrícula de las carreras asociadas y como consecuencia directa en la cantidad de titulaciones universitarias.

Es importante destacar que uno de los objetivos específicos de la Ley 7169 Promoción de desarrollo científico y tecnológico de nuestro país (Gobierno de la República de Costa Rica, 1990) es "establecer estímulos e incentivos para los sectores privado, público y para las instituciones de educación superior universitaria y otros centros de educación, con la finalidad de que incremente la capacidad de generar ciencia y tecnología y de que éstas puedan articularse entre sí (p. 1); sin embargo, entre los señalamientos realizados por el personal consultado mencionan que se debe invertir en educación e investigación ya que estos permiten el fortalecimiento de las uniones entre las industrias y los centros de educación superior.

En síntesis, se puede plantear que las principales necesidades que se deben afrontar son: incrementar la inversión en infraestructura y educación, potenciar las vocaciones científicas y aumentar las investigaciones en este campo. Para lograr esto, es necesario modernizar la estructura productiva, la maquinaria y el equipamiento y, por supuesto, incrementar el capital, para así competir dentro de los mercados mundiales.

Sobre cuáles proyectos son necesarios para potenciar el campo científico-tecnológico de Costa Rica, las personas expertas opinan que deben ser proyectos innovadores en este campo, además de permitir la interacción entre el sector público y privado. Con respecto a esto, el MICIT (2011) menciona que existe una correlación estable y duradera entre las inversiones en innovación y el crecimiento de la productividad de los países, por lo tanto, la relación de casualidad va de la innovación hacia mayor productividad y crecimiento, y no al revés. Es por esto que la innovación debe ser un elemento crucial para desarrollar el país en ciencia y tecnología.

Otro aspecto que consideran de vital importancia es la educación, por medio de la cual se podría mejorar la promoción de la ciencia y la tecnología, como lo dice Erazo (2008), el logro de los estándares de calidad en la educación científica y tecnológica son factores claves para el desarrollo económico y social de un país y estos se encuentran articulados con las política educativas y las científico-tecnológicas. De ahí que aquellos países que han articulado estas políticas son los que hoy en día han logrado tener grandes avances en este campo.

En el Plan Nacional de Desarrollo 2011-2014 "María Teresa Obregón Zamora" (Gobierno de la República de Costa Rica, 2010) la educación en Costa Rica siempre ha sido un motor 
doi: http://dx.doi.org/10.15359/ree.20-1.2

URL: http://www.una.ac.cr/educare

CORREO: educare@una.cr

fundamental del desarrollo, y a nivel latinoamericano el país se ha destacado por dar grandes saltos en los ejes económico, social y ambiental. En concordancia con esto, las opiniones de las personas expertas apuntan a que el campo educativo es el pilar para promover el desarrollo científico-tecnológico: es este el que potencializa la parte económica y social.

Sin embargo, personal experto de la ANC, Fundación CIENTEC y del MICITT, coinciden en que la educación secundaria que se imparte en el país no está desarrollando las dimensiones de la competencias científicas-tecnológicas, asimismo, consideran que se han dejando de lado modelos que permitirían la formación de competencias que contribuirían a solventar las necesidades de desarrollo científico y tecnológico del país. Por su parte, del personal experto COMEX, CONICIT e INTEL, también consideran que el impulso de estas competencias se ha ido desarrollando de forma limitada, debido a que la estructura educativa y la labor docente no se adecúan a las necesidades del país en el campo de la ciencia y la tecnología.

En esta misma línea, las asesorías de Biología, Física y Química del MEP piensan que es necesario un cambio para que se promuevan, en estudiantes de secundaria, destrezas y habilidades que contribuyan al desarrollo científico-tecnológico; aducen que los programas de estudio deben ser diferentes para las distintas modalidades educativas existentes; que es urgente dotar de insumos tecnológicos que faciliten el desarrollo de las clases para buscar respuestas coherentes a los cambios rápidos del entorno; que hay que promover las vocaciones científicas, la curiosidad; que se debe fortalecer la investigación y desarrollar actividades motivadoras e interesantes para inducir al aprendizaje de las áreas científicas y tecnológicas. En concordancia con lo anterior, Vázquez y Manassero (2009) plantean la necesidad de que la educación científicotecnológica promueva aspectos actitudinales, afectivos y emocionales, que logren generar curiosidad y motivar al estudiantado hacia el estudio de estas áreas del conocimiento.

El Programa Estado de la Nación (2011) propone mejorar la calidad de la educación científica afianzando los programas exitosos, como los colegios científicos y las iniciativas relacionadas con la metodología de la enseñanza de las ciencias basada en la indagación, debido a que este enfoque ha cosechado buenas experiencias a nivel internacional, así mismo, destaca que es importante contar con una política nacional que establezca lineamientos con el desarrollo y seguimiento de la formación científica en todo el sistema educativo.

Sobre la implementación de una cultura científica que permita el desarrollo en este campo, las personas expertas en educación opinan que existen algunas limitantes relacionadas con la falta de materiales didácticos y espacios que faciliten una enseñanza que promueva el conocimiento relacionado con la realidad vivida y que, además, fomente una enseñanza por indagación; así mismo, consideran que falta un mayor número de docentes con la preparación y capacitación debidas. Por otra parte, falta invertir más recursos en la educación secundaria y dar una mejor redistribución del tiempo en las asignaturas de ciencias. 
doi: http://dx.doi.org/10.15359/ree.20-1.2

Según Vázquez, Acevedo y Manassero (2005), la frustración del estudiantado ante la ciencia escolar se debe a causas bien conocidas como: currículos recargados; contenidos difíciles y aburridos; profesorado poco innovador para incorporar, a la educación científica, mejoras en la metodología; imagen estereotipada del ámbito científico-tecnológico y de los científicos y científicas, entre otras; por lo que se debe resaltar la presencia y la aportación de personal docente con perfiles diferentes que complementen la educación científica formal.

Además, las personas expertas opinan que, en el planteamiento actual de la educación científica, existe desarticulación porque el currículo es de carácter independiente para cada asignatura, cuando debería ser integrado en las tres áreas: la visión fragmentada con la que se asumen los programas hace que las temáticas no se articulen entre las disciplinas para conformar espacios comunes de trabajo, razón por la cual aun cuando ciencias es el área que más número de lecciones tiene en el plan curricular actual del ciclo diversificado, no se aprovecha esto para el desarrollo de un abordaje pedagógico coherente con la naturaleza de la disciplina, por lo que se han hecho persistentes quejas de la deficiente formación de estudiantil para asumir estudios universitarios en cada una de las áreas de las ciencias (Alfaro y Villegas, 2010).

El país debe potenciar las disciplinas del área científico-tecnológica, porque estas son importantes para el desarrollo en este campo, ya que la tecnología en todas partes del mundo, según la Organización Internacional del Trabajo (OIT, 2008, párr. 1):

Se presenta como una fuerza potente capaz de transformar la vida social, económica y política. En muchos casos, el desarrollo continuo y la aplicación de la tecnología han creado un amplio y novedoso espectro de oportunidades económicas y de empleo, [permitiendo que muchos países en desarrollo aceleren este proceso].

Acerca de los tipos de modelos educativos que es necesario fomentar para satisfacer las necesidades de desarrollo CIENTÍfICO Y TECNOLÓGICO del país, coinciden en que se deben potenciar modelos que respondan a las necesidades de desarrollo, como el modelo constructivista que fomenta un ambiente experimental o un modelo exploratorio que oriente y potencie el interés estudiantil, como se hace actualmente en las modalidades técnicas.

En relación con lo anterior, es fundamental destacar que, según Aguerrondo (2009), la crisis del modelo científico está llevando a una redefinición muy profunda de los modos de hacer ciencia y, sobre todo, del conocimiento dentro de la sociedad con base en la idea de que los cambios que tienen lugar en el pensamiento científico contemporáneo modifican la perspectiva del saber y los ideales de racionalidad, por lo que, como consecuencia, se ha dado un cambio en la ciencia y la vida cotidiana, al formularse problemas nuevos que producen una revolución en el saber. Se puede afirmar que las ciencias y la tecnología han terminado por transformar numerosos espacios de las sociedades contemporáneas; sin embargo, a pesar 
doi: http://dx.doi.org/10.15359/ree.20-1.2

URL: http://www.una.ac.cr/educare

CORREO: educare@una.cr

de que son innegables los beneficios que de tal transformación se obtienen, también son numerosos los riesgos que han surgido de tan vertiginoso desarrollo; lo cual obliga a que la ciencia y la tecnología deban ser vistas con una actitud más crítica y reflexiva (Osorio, 2002).

La educación científica para hacer frente a las demandas actuales de la sociedad debe responder a un aprendizaje que fomente capacidades y habilidades y que, con base en la investigación, se logre sembrar el interés por las vocaciones científicas. De acuerdo con Rebollo (2010), llevar a cabo en las aulas una enseñanza basada en el desarrollo de competencias supone una innovación educativa de gran calado que requerirá esfuerzo y atención de múltiples factores, por lo que, la introducción del término y su aplicación como elemento curricular puede y debe ser una magnífica ocasión para lograr una educación científica de mayor calidad donde el estudiantado adquiera los aprendizajes básicos para utilizarlos de manera efectiva en distintas situaciones y contextos.

Es necesario que el estudiantado, en el proceso de su formación, tenga ocasión de afrontar auténticos problemas científicos y ensaye las estrategias de solución que contribuyen a ampliar el conocimiento; ya que los problemas científicos como actividad escolar tienen la facultad de contribuir al desarrollo competencial de resolución de problemas, como lo hacen a menudo los científicos y científicas en el mundo real. Por tanto, para aprender hay que problematizar los ejercicios y actividades que se plantean en la clase que permitan construir los conocimientos que se consideran fundamentales ya sea para desempeñarse como profesionales competentes en el campo de las ciencias o bien para ser una ciudadanía responsable y alfabetizada científicamente (Quintanilla et al., 2010).

Para las personas expertas en educación, los programas de estudio de las disciplinas de la ciencia permiten, en algún grado, el desarrollo de competencias científicas; sin embargo, para que estén en concordancia con el desarrollo científico y tecnológico del país, es necesario diferenciarlos según la modalidad de colegio y que el personal docente promueva, dentro de los recintos educativos, dimensiones y capacidades que les permitan, al estudiantado que egresa, tener una serie de competencias para desarrollar una vocación científica-tecnológica.

Según su perspectiva, dentro de las competencias científicas deseables que debe adquirir un ciudadano o ciudadana que egresa de secundaria están: responder a un pensamiento innovador que le permita resolver problemas referentes al ámbito científico-tecnológico, implementar el método científico en diferentes situaciones de la vida diaria, tener capacidad de desarrollar investigaciones $y$, a su vez, que sean personas emprendedoras, entre otros aspectos.

Cabe destacar que la formación de competencias resulta ser un proceso constructivo, socializado, que solo es posible en un espacio interdisciplinar partiendo de una concepción participativa, con el convencimiento de que el contenido es socialmente construido e históricamente desarrollado. Por consiguiente, las competencias indispensables en la 
doi: http://dx.doi.org/10.15359/ree.20-1.2

ciudadanía se alcanzan mediante un proceso en el que se trabaja de manera interrelacionada entre los núcleos de conocimientos, las habilidades generalizadas y los valores profesionales y sociales, donde lo interdisciplinario se debe manifestar en lo académico, lo investigativo y lo laboral (Valera, 2010).

Es decir, para alcanzar mejoras en el desarrollo de competencias en los estudiantes y las estudiantes es necesario minimizar las limitaciones que se han venido adquiriendo con el transcurrir del tiempo en el marco educativo. Las personas expertas en educación mencionan que se deben hacer las clases más prácticas y vivenciales, esto porque si bien el conocimiento científico se justifica según el contexto de aplicación y de utilización del saber, la enseñanza de las ciencias debe favorecer la adquisición de una actitud en la cual el asombro, la confianza en sí y el espíritu crítico permitan una extensión de las facultades y habilidades necesarias en la sociedad (Ministerio de Educación y Cultura [MEC], 2012).

Además, mencionan la necesidad de integrar los contenidos, no solo a nivel de programas sino también en el trabajo de aula, lo cual tiene como consecuencia que el educando sea capaz de crear relaciones entre distintas disciplinas, buscando así un ser más integral. Y esto es constatado por Lazo (2011), al decir que no se puede pensar en el funcionamiento de un país sin que el desarrollo científico social que se presenta en las instituciones educativas sea interdisciplinariamente, ya que con esto se piensa, se educa e se instruye en una misma línea.

\section{Conclusiones}

- $\quad$ El programa de estudio de Biología es el que en mejor medida demuestra las dimensiones (conocimiento del saber y del saber hacer, capacidad, actitud y contexto) de las competencias evidenciadas en los objetivos a través de las capacidades esperadas a corto plazo, de los cuales se desprende la articulación de estas dimensiones en cada unidad temática del programa de estudios, aspecto que es determinante para que la educación media contribuya con el desarrollo científico y tecnológico del país.

- Con respecto a la presencia de las dimensiones en los objetivos de los programas, se encontró que la dimensión actitud es la que se encuentra en menor proporción y la de capacidad es la que tiene mayor presencia en las tres áreas científicas, sin embargo, la más promovida por docentes de Biología, Física y Química de las modalidades académica, científica y técnica es precisamente la de actitud.

- $\quad$ Otra dimensión que se promueve es la de conocimiento que la trabajan utilizando las metodologías tradicionales, basadas en la lectura del libro de texto, fotocopias y explicaciones en la pizarra con la instrucción docente directa.

- $\quad$ Acerca de la dimensión contexto, en términos generales se encontró que es la que los docentes (de la muestra en estudio) promueven en menor medida en sus clases. De lo 
doi: http://dx.doi.org/10.15359/ree.20-1.2

URL: http://www.una.ac.cr/educare

CORREO: educare@una.cr

anterior se deduce que dentro de las aulas existe una descontextualización de lo que se imparte y las necesidades de desarrollo que persiguen las políticas en este campo en el país, porque hay una desvinculación entre el currículo pretendido en el programa de estudio y el contexto.

- $\quad$ Con respecto a la opinión de las personas expertas, en el ámbito de desarrollo científicotecnológico hay consenso de que la educación en las disciplinas científicas impartidas en el país a nivel de secundaria no están desarrollando las dimensiones de la competencias científicas-tecnológicas que permitan la formación de las capacidades necesarias para pensar en un desarrollo científico acorde con las políticas nacionales; asimismo, consideran que se ha dejado de lado modelos que permitirían la formación de competencias por medio de las cuales se podría mejorar la promoción de la ciencia y la tecnología.

- $\quad$ Además, indican que el país promueve una educación científica que no está del todo enfocada en las necesidades reales de desarrollo. Aducen que, sumado a esto, se encuentra la falta de infraestructura, poco material didáctico, escaso equipo de laboratorio, docentes casi sin acceso a actualización y la falta un currículo que esté en concordancia con las políticas de desarrollo del país, entre otros aspectos.

- Por otro lado, señalan la falta de vocaciones científicas como un factor que incide directamente sobre el desarrollo de un país cuando de ciencia y tecnología se trata, mencionan que deben existir mecanismos para incrementar el recurso humano en estas áreas y la educación debe ser uno de ellos.

- $\quad$ Por otra parte piensan que es necesario promover en el estudiantado de secundaria destrezas y habilidades que contribuyan al desarrollo científico-tecnológico. Además, plantean que los programas de estudio para las distintas modalidades educativas deben ser diferentes y que es urgente dotar de insumos tecnológicos que faciliten el desarrollo de las clases para buscar respuestas coherentes a los cambios rápidos del entorno; así como fortalecer la investigación y desarrollar actividades que interesen y motiven al estudiantado al aprendizaje de las áreas científicas y tecnológicas.

- Con los resultados obtenidos se determinó que de la muestra en estudio, los docentes del colegio científico son los que promueven en mayor medida las dimensiones presentes en los objetivos; sin embargo, no se encontró que exista relación entre lo que se enseña en la clase y las políticas de desarrollo científico del país.

- Para hacer frente a las demandas actuales, un aspecto medular es que los programas de la educación científica se planteen por competencias y estén relacionados con las políticas de desarrollo científico-tecnológico del país, para que responda a un aprendizaje que fomente capacidades y habilidades que permitan dar solución a situaciones cotidianas con conocimientos científicos. 
doi: http://dx.doi.org/10.15359/ree.20-1.2

URL: http://www.una.ac.cr/educare

\section{Referencias}

Aguerrondo, I. (2009). Conocimiento complejo y competencias científicas (Series IBE Working Papers on Curriculum ISSues 8). Ginebra, Suiza: UNESCO-IBE. Recuperado de http://www.ibe. unesco.org/fileadmin/user upload/Publications/Working Papers/knowledge compet ibewpci 8.pdf

Alfaro, G. y Villegas, L. R. (2010). La educación científica en Costa Rica. Ponencia presentada para el Tercer informe estado de la educación. San José, Costa Rica: Programa Estado de la Nación. Recuperado de http://www.estadonacion.or.cr/files/biblioteca virtual/educacion/003/ Alfaro Villegas 2010 Educacion cientifica.pdf

Arnau, L. y Montané, J. (Diciembre, 2010). Aportaciones sobre la relación conceptual entre actitud y competencia, desde la teoría del cambio de actitudes. Electronic Journal of Research in Educational Psychology, 8(22), 1283-1302. Recuperado de http://www.investigacionpsicopedagogica.org/revista/new/ContadorArticulo.php?465

Cano, M. E. (2008). La evaluación por competencias en la educación superior. Profesorado. Revista de curriculum y formación del profesorado, 12(3), 1-16. Recuperado de http://www. ub.edu/cubac/sites/default/files/la evaluacion por competencias en la educacion superior $0 . p d f$

Carrasco, S. (2010). Reseña [Paradojas y dilemas de las universidades iberoamericanas ante la sociedad del conocimiento, por A. Gewrc (Coord.)]. Revista de Universidad y Sociedad del Conocimiento, 7(1), 1-7. Recuperado de http://dialnet.unirioja.es/servlet/ articulo?codigo $=3119051$

Ceballos, Á. (2004). La escuela tradicional. San Luis Potosí, México: Universidad Abierta.

Ciarli, T. y Giuliani, E. (2005). Inversión extranjera directa y encadenamientos productivos en Costa Rica. En M. Cimoli (Ed.), Heterogeneidad estructural, asimetrías tecnológicas y crecimiento en América Latina (pp. 127-157). Santiago de Chile: Naciones Unidas. Recuperado de http:// www.cepal.org/iyd/noticias/paginas/4/31434/W35 CIMOLI.pdf

Cordón, R. (2008). Enseñanza y aprendizaje de procedimientos científicos (contenidos procedimentales) en la educación secundaria obligatoria: Análisis de la situación, dificultades y perspectivas (Tesis doctoral). Universidad de Murcia. Recuerado de https:// digitum.um.es/jspui/bitstream/10201/3613/1/CordonAranda.pdf

De Freitas Dias de Souza, K. A. y Alves, A. (2010). Reflexiones sobre el papel de la contextualización en la enseñanza de ciencias. Enseñanza de las Ciencias, 28(2), 275-284. Recuperado de http://ensciencias.uab.es/issue/view/1 
doi: http://dx.doi.org/10.15359/ree.20-1.2

URL: http://www.una.ac.cr/educare

CORREO: educare@una.cr

Erazo, M. S. (2008). Elementos para un debate sobre las relaciones entre educación, sociedad y desarrollo científico y tecnológico, en las universidades del Estado. Cuadernos de Docencia Universitaria, 1(1), 150-160. Recuperado de http://www.educandus.cl/ojs/index.php/ cdocencia/article/viewFile/17/19

García-Carmona, A. y Criado, A. M. (2010). La competencia social y ciudadana desde la educación científica: Una experiencia en torno a la energía nuclear. Investigación la Escuela, 71, 25-38. Recuperado de https://idus.us.es/xmlui/bitstream/handle/11441/25974/file 1. pdf? sequence $=1$ \&isAllowed $=y$

Gobierno de la República de Costa Rica. (1990). Ley 7169. Promoción del desarrollo científico y tecnológico. San José, Costa Rica: Gobierno de la República de Costa Rica. Recuperado de http://www.pgrweb.go.cr/scij/Busqueda/Normativa/Normas/nrm texto completo.aspx? param $1=$ NRTC\&nValor $1=1 \&$ nValor $2=11908 \&$ nValor $3=91174 \&$ strTipM $=$ TC

Gobierno de la República de Costa Rica. (2010). Plan nacional de desarrollo 2011-2014 "María Teresa Obregón Zamora". San José, Costa Rica: MIDEPLAN. Recuperado de http:// documentos.mideplan.go.cr/alfresco/d/d/workspace/SpacesStore/122fcd1c-53a7-47a7a0ad-84cac6f1d7b9/PND-2011-2014-Maria-Teresa-Obregon-Zamora.pdf

Gobierno Vasco, Universidades e Investigación. (2009). Competencia en cultura científica, tecnológica y de la salud: Educación secundaria obligatoria. País Vasco: Autor. Recuperado de http://www.hezkuntza.ejgv.euskadi.eus/r43-573/es/contenidos/ informacion/dig publicaciones innovacion/es curricul/adjuntos/14 curriculum competencias 300/300007c Pub BN Competencia Cientifica ESO c.pdf

Gobierno Vasco, Universidades e Investigación. (2011). PISA: Competencia científica para el mundo del mañana: I. Marco y análisis de los ítems (Proyecto de evaluación internacional del alumnado de 15 años). País Vasco: ISEI.IVEI. Recuperado de http://www.isei-ivei.net/ cast/pub/itemsliberados/Ciencias2011/ciencias PISA2009completo.pdf

González, C., Martínez, M. T., Martínez, C., Cuevas, K. y Muñoz, L. (2009). La educación científica como apoyo a la movilidad social: Desafíos en torno al rol del profesor secundario en la implementación de la indagación científica como enfoque pedagógico. Estudios Pedagógicos, 35(1), 63-78. doi: http://dx.doi.org/10.4067/50718-07052009000100004

Guadamuz, L. (2005). La Costa Rica del siglo XXI: Un desarrollo basado en la educación y el conocimiento. San José, Costa Rica: Fundación Crusa. Recuperado de http://aplicaciones02. fod.ac.cr/igccire/index.php?option=com remository\&ltemid=40\&func=startdown\&id=1 09\&lang=es 
doi: http://dx.doi.org/10.15359/ree.20-1.2

URL: http://www.una.ac.cr/educare

Hernández, S. y Zacconi, F. C. (septiembre de 2010). Competencias básicas: Alfabetización científica en química al alcance de todos. Comunicación presentada en el Congreso Iberoamericano de Educación, Metas 2021. Buenos Aires, Argentina. Recuperado de http://www.chubut.edu.ar/descargas/secundaria/congreso/COMPETENCIASBASICAS/ RLE3304 Hernandez.pdf

Instituto de Tecnologías Educativas (2010). Habilidades y competencias del siglo XXI para los aprendices del nuevo milenio en los países de la OCDE. París: Autor. Recuperado de https:// docs.google.com/document/preview?hgd=1\&id=181BFFB2Y51xGLkLMwEI6X5BKRkOW vSIp3Z83RwSP2Fo\&pli=1

Instituto Tecnológico y de Estudios Superiores de Monterrey. (2009). Módulo 5 Inversión en infraestructura para el desarrollo económico y social. México: Autor. Recuperado de http:// www.cca.org.mx/funcionarios/cursos/ap089 2/apoyos/m5/modulo5 vi.pdf

Jabif, L. (2007). Orientaciones prácticas para docentes: La docencia universitaria bajo un enfoque de competencias. Valdivia, Chile: Universidad Austral de Chile. Recuperado de http://ciuf. unsaac.edu.pe/sisciuf/upload doc/DP-20131126-120334.pdf

Lazo, M. A. (Mayo, 2011). La interdisciplinariedad y la integralidad una necesidad de los profesionales de la educación. Revista Cuadernos de Educación y Desarrollo. 3(27). Recuperado de http://www.eumed.net/rev/ced/27/malp.htm

Lemke, J. L. (2006). Investigar para el futuro de la educación científica: Nuevas formas de aprender, nuevas formas de vivir. Enseñanza de las Ciencias, 24(1), 5-12. Recuperado de http://www.raco.cat/index.php/Ensenanza/article/view/73528/84736

López, J. (Noviembre de 2006). Las competencias básicas del currículo en la LOE. Documento presentado en el V Congreso Internacional "Educación y Sociedad". Granada, España. Recuperado de http://www.redes-cepalcala.org/inspector/DOCUMENTOS\%20Y\%20 LIBROS/COMPETENCIAS/LAS\%20COMPETENCIAS\%20BASICAS\%20EN\%20LA\%20LOE.pdf

Macaya, G. (2006). Algunos indicadores sobre la actividad científica y tecnológica. En G. Macaya y A. Cruz (Comps.), Proyecto estrategia siglo XXI. Visión de la ciencia y la tecnología en Costa Rica: Una construcción colectiva (pp. 9-17). San, José, Costa Rica: Fundación Costa Rica Estados Unidos de América para la Cooperación. Recuperado de http://www.estrategia. cr/content/images/pdfs/tomo2.pdf

Ministerio de Ciencia y Tecnología (MICIT).(2011). Plan nacional de ciencia, tecnología e innovación 2011-2014. San José, Costa Rica: Autor. Recuperado de http://www.conicit.go.cr/servicios/ listadocs/programas-nac-cyt/Plan Nacional CTI-2011-2014.pdf 
doi: http://dx.doi.org/10.15359/ree.20-1.2

URL: http://www.una.ac.cr/educare

CORREO: educare@una.cr

Ministerio de Ciencia, Tecnología y Telecomunicaciones (MICITT). (2012). Indicadores nacionales 2010-2011. Ciencia, tecnología e innovación Costa Rica. San José, Costa Rica: Autor. Recuperado de http://www.conicit.go.cr/servicios/listadocs/IndicadoresCyT-2009.pdf

Ministerio de Educación. (2009). Educación científica "ahora": El informe Rocard. España: Secretaría General Técnica.

Ministerio de Educación (MEDUCA). (2012). Orientaciones para la aplicación de competencias en el aula (Serie: Hacia un currículo en competencia № 1). Ciudad de Panamá, Panamá: Autor. Recuperado de http://consulta.meduca.gob.pa/04unad/DNCYTE/docs/HAClA UN CURRICULO POR COMPETENCIA/1.pdf

Ministerio de Educación Pública de Costa Rica. (2005). Programa de Estudio de Biología Educación Diversificada. San José, Costa Rica: Autor. Recuperado de http://www.mep.go.cr/sites/ default/files/programadeestudio/programas/biologia.pdf

Ministerio de Educación Pública de Costa Rica. (2003). Programa de Estudio de Física Educación Diversificada. San José, Costa Rica: Autor. Recuperado de http://www.mep.go.cr/sites/ default/files/descargas/programas-de-estudio/fisica.pdf

Ministerio de Educación Pública de Costa Rica. (s. f.). Programa de Estudio de Química para la Educación Diversificada. San José, Costa Rica: Autor. Recuperado de http://www.mep. go.cr/sites/default/files/descargas/programas-de-estudio/quimica.pdf

Ministerio de Educación y Cultura (MEC). (2012). Semana de la ciencia y la tecnología en Uruguay 2012: Informe $7^{a}$ Semana de la Ciencia y la Tecnología. Montevideo, Uruguay: La imprenta. Recuperado de http://www.semanacyt.org.uy/uploads/historial/1392313337 ffuyt.pdf

Organización de Estados Iberoamericanos para la Educación, la Ciencia y la Cultura (OEI). (2012). Ciencia, tecnología e innovación para el desarrollo y la cohesión social. Programa iberoamericano en la década de los bicentenarios: Madrid: Autor. Recupeado de http:// www.oei.es/documentociencia.pdf

Organización Internacional del Trabajo (OIT). (2008). Competencias y emprendimiento: Reducir la brecha tecnológica y las desigualdades de género. Ginebra, Suiza: Autor. Recuperado de http://www.ilo.org/wcmsp5/groups/public/@dgreports/@gender/documents/ publication/wcms 101145.pdf

Organización para la Cooperación y el Desarrollo Económico (OCDE). (2006). PISA 2006. Marco de la evaluación: Conocimientos y habilidades en ciencias, matemáticas y lectura. Madrid: Santillana. Recuperado de http://www.oecd.org/pisa/39732471.pdf 
doi: http://dx.doi.org/10.15359/ree.20-1.2

URL: http://www.una.ac.cr/educare

Osorio, C. (Enero-Abril, 2002). La educación científica y tecnológica desde el enfoque en ciencia, tecnología y sociedad. Aproximaciones y experiencias para la educación secundaria. Revista Iberoamericana de Educación: 28, 61-81. Recuperado de http://www.rieoei.org/ rie28f.htm

Pedrinaci, E. (2011). ¿Qué ciencia enseñar? Entre el currículo y la programación del aula. En P. Cañal (Coord.), Didáctica de la biología y la geología: Formación y desarrollo profesional del profesorado (pp. 49-70). Barcelona: Graó.

Programa del Estado de la Nación. (2011). Decimoséptimo informe del Estado de la nación en desarrollo humano sostenible. San José, Costa Rica: Autor. Recuperado de http://www. estadonacion.or.cr/estado-nacion/nacion-informes-anteriores/informes-2001-2011/xviiinforme2011

Quintanilla, M., Joglar, C., Jara, R., Camacho, J., Ravanal, E., Labarrere, A. ... Chamizo, J. (2010). Resolución de problemas científicos escolares y promoción de competencias de pensamiento científico: ¿Qué piensan los docentes de química en ejercicio? Enseñanza de las Ciencias, 28(2), 185-198. Recuperado de Enseñanza de las Ciencias. Recuperado de http://ensciencias.uab.es/article/view/315/pdf

Rebollo, M. (enero de 2010). Análisis del concepto de competencia científica: Definición y dimensiones. Ponencia presentada en el I Congreso de inspección de Andalucía: Competencias básicas y modelos de intervención en el aula. Junta de Andalucía, Consejería de Educación, Málaga, España. Recuperado de http://redes-cepalcala.org/inspector/ DOCUMENTOS\%20Y\%20LIBROS/COMPETENCIAS/I\%20CONGRESO\%20INSPECCION\%20 ANDALUCIA/downloads/rebollo.pdf

Salas, R. E. (2008). Estilos de aprendizaje a la luz de la neurociencia. Bogotá: Cooperativa Editorial Magisterio.

Sanmartí, N. (2008) ¿Qué conlleva desarrollar la competencia científica? Barcelona: Universidad Autónoma de Barcelona. Recuperado de http://www.mrpmenorca.cat/index2. php?option=com docman\&task= doc view\&gid=116\&

Torres, M. I. (Enero-Junio, 2010). La enseñanza tradicional de las ciencias versus las nuevas tendencias educativas. Revista Educare, 14(1), 131-142. Recuperado de http://www. revistas.una.ac.cr/index.php/EDUCARE/article/view/1515

Valera, R. (Enero-Junio, 2010). El proceso de formación del profesional en la educación superior basado en competencias: El desafío de su calidad, en busca de una mayor integralidad de los egresados. Civilizar, 10(18), 117-134. Recuperado de http://www.usergioarboleda.edu. co/civilizar/civilizar-18/El\%20proceso\%20de\%20formacion....pdf 
doi: http://dx.doi.org/10.15359/ree.20-1.2

URL: http://www.una.ac.cr/educare

CORREO: educare@una.cr

Vázquez, Á.y Manassero, M. A. (2009). Expectativas sobre un trabajo futuro y vocaciones científicas en estudiantes de educación secundaria. Revista Electrónica de Investigación Educativa, 11(1): 1-20. Recuperado de http://www.scielo.org.mx/pdf/redie/v11n1/v11n1a3.pdf

Vázquez-Alonso, A., Acevedo-Díaz, J. A. y Manassero, M. A. (2005). Más allá de la enseñanza de las ciencias para científicos: Hacia una educación científica humanística. Revista Electrónica de Enseñanza de las Ciencias, 4(2), 1-30. Recuperado de http://reec.uvigo.es/volumenes/volumen4/ ART5 Vol4 N2.pdf

Zúñiga, A., Leiton, R. y Naranjo, J. A. (2011). Nivel de desarrollo de las competencias científicas en estudiantes de secundaria de (Mendoza) Argentina y (San José) Costa Rica. Revista Iberoamericana de Educación, 56(2): 1-12. Recuperado de http://www.rieoei.org/ deloslectores/4246Zuniga.pdf

\section{(9) Cómo citar este artículo en APA:}

Padilla-Canales, C., Brooks-Calderón, P., Jiménez-Porras, L. D. y Torres-Salas, M. I. (Enero-abril, 2016). Dimensiones de las competencias científicas esbozadas en los programas de estudio de Biología, Física y Química de la Educación Diversificada y su relación con las necesidades de desarrollo científico-tecnológico de Costa Rica. Revista Electrónica Educare, 20(1), 1-26. doi: http://dx.doi.org/10.15359/ree.20-1.2

Nota: Para citar este artículo en otros sistemas puede consultar el hipervínculo "Como citar el artículo" en la barra derecha de nuestro sitio web: http://www.revistas.una.ac.cr/index.php/EDUCARE/index 